\title{
Mesoscopic Simulation for Self-organization in Surface Processes
}

\author{
David J. Horntrop \\ New Jersey Institute of Technology, \\ Department of Mathematical Sciences, \\ Newark, NJ 07102 USA
}

\begin{abstract}
The self-organization of particles in a system through a diffusive mechanism is known as Ostwald ripening. This phenomenon is an example of a multiscale problem in that the microscopic level interaction of the particles can greatly impact the macroscale or observable morphology of the system. The mesoscopic model of this physical situation is a stochastic partial differential equation which can be derived from the appropriate particle system. This model is studied through the use of recently developed and benchmarked spectral schemes for the simulation of solutions to stochastic partial differential equations. The results included here demonstrate the effect of adjusting the interparticle interaction on the morphological evolution of the system at the macroscopic level.
\end{abstract}

\section{Introduction}

The coarsening phenomenon by which mass is transported via diffusion in order to reduce the overall interfacial area is known as Ostwald ripening. In general, larger droplets grow at the expense of smaller droplets-the number of droplets decreases while the average size of the remaining droplets increases because of the conservation of mass in the system. This phenomena has received a great deal of study in the literature from theoretical, experimental, and computational points of view. ([1], [2], and [3] are just a few examples.) However, Ostwald ripening is also a very difficult problem to study due to the inherently multiscale nature of the problem. In this note, a mesoscopic model which can be related to many of the commonly studied models of phase separation is used in a computational study of domain coarsening. To begin, the model under consideration here will be described.

\section{Description of Mesoscopic Model}

Mesoscopic (or local mean field) models are designed to bridge the gap between microscopic (molecular) and macroscopic (observable) scales by incorporating microscopic level behavior in the macroscopic level. Mesoscopic models can be 
derived through a coarse graining of the underlying microscopic system in a rigorous fashion without the introduction of artificial cutoffs. Details of the derivation of mesoscopic models are given in [4], [5], and [6]. Here, this modeling approach will be briefly described and the mesoscopic model appropriate for studying Ostwald ripening will be given.

In the mesoscopic framework, each micromechanism is incorporated through an evolution equation of the average coverage which is derived from the specific micromechanism under consideration. These evolution equations contain stochastic forcing terms as a result of a direct derivation rather than being included in an ad hoc manner as a means of describing microscale behavior. Thus, the mesoscopic model equations are stochastic partial integrodifferential equations which incorporate the molecular interactions explicitly through convolutions.

The mesoscopic model that would be an appropriate model of Ostwald ripening arises from the spin exchange (surface diffusion) mechanism and can be given as follows:

$$
u_{t}-D \nabla \cdot\left[\nabla u-\beta u(1-u) \nabla J_{m} * u\right]+\gamma \operatorname{div}[\sqrt{2 D u(1-u)} d W(x, t)]=0
$$

where $u$ is the concentration of the particles on the surface, $D$ is the diffusion constant, $\beta$ is proportional to the inverse of the temperature of the underlying Ising model, $J_{m}$ is the migration potential, $\gamma$ is proportional to the interaction length of the particles, and $d W$ represents a process that is delta correlated (white noise) in both space and time $\langle d W(x, t)\rangle=0$ and $\left\langle d W(x, t) d W\left(x^{\prime}, t^{\prime}\right)\right\rangle=\delta\left(x-x^{\prime}\right) \delta\left(t-t^{\prime}\right)$ where the angular brackets are used to denote mean values. It is important to observe that the noise in (1) is multiplicative rather the additive noise that is commonly added to deterministic models in an ad hoc manner.

\section{Description of Simulation Method}

The numerical scheme that is used to study the mesoscopic model in (1) is based upon a generalization of spectral schemes for deterministic partial differential equations to the stochastic setting. Spectral schemes are a particularly attractive way to study (1) due to the simplicity of calculating convolutions spectrally. Just as in the deterministic setting, spatial variables and derivatives are treated spectrally while the time evolution is calculated using finite differences. A detailed description of this scheme is given in [7] where some exactly solvable problems are used as computational benchmarks to validate this method. The emphasis here will be on those aspects of the scheme which are unique to the stochastic system, such as the spectral treatment of the multiplicative noise term in (1) and the use of a suitable time discretization technique. For the sake of simplicity in the description of the spectral method in this section, it will be assumed that the stochastic partial differential equation has only one spatial dimension. The method straightforwardly generalizes to higher spatial 
dimension as evidenced by the two spatial dimension results presented in Section 4.

\subsection{Treatment of Noise Term}

One of the most important steps for developing a spectral scheme for a stochastic partial differential equation such as the mesoscopic model in (1) is to determine a spectral representation for the spatial component of the noise term. It is wellknown in the stochastic processes literature that a stationary, isotropic, Gaussian random field $v(x)$ can be represented by the following stochastic integral in Fourier space:

$$
v(x)=\int e^{2 \pi i x \cdot \xi} S^{\frac{1}{2}}(\xi) d W(\xi)
$$

where $W$ is Brownian motion and $S$ is the spectral density function of the random field. Most consistent numerical schemes are based upon discretizations of this stochastic integral $([8],[9])$. The simplest such discretization uses equispaced nodes and is known as the Fourier method and is essentially a Fourier series. This approximation is

$$
v(x) \approx \sum_{j=1}^{M} a_{j} \cos \left(2 \pi \xi_{j} x\right)+b_{j} \sin \left(2 \pi \xi_{j} x\right)
$$

where $a_{j}$ and $b_{j}$ are independent Gaussians with mean zero and variance $S\left(\xi_{j}\right) \Delta \xi$. Due to the periodicity of the approximation in (3), the Fourier method would not be suitable in applications in which the desired random field has long range correlations [8]. However, since the random field that is needed for the numerical study of (1) has delta correlation (lacks long range correlations), the Fourier method should give a good approximation. The Fourier method is also computationally attractive since an FFT can be used directly to evaluate (3) at all physical space lattice sites.

The next issue in the treatment of the noise term in (1) is determining how to use (3) to obtain a spectral representation of the multiplicative noise term. Because each realization of the noise at a given time is approximated by a Fourier series representation (3), the noise term in (1) can be calculated by completing all multiplications in physical space and all differentiations in Fourier space, passing back and forth between physical space and Fourier space as necessary using an FFT. Thus, the existence of the Fourier series representation of the noise allows the treatment of the stochastic term in the same basic fashion as for deterministic terms in partial differential equations.

\subsection{Time Discretization}

At this point, the solution of the stochastic partial differential equation in (1) effectively has been reduced to the solution of a stochastic ordinary differential equation which can be written in the form

$$
u_{t}=a(u) d t+b(u) d W(t)
$$


where $a$ is the drift coefficient and $b$ is the diffusion coefficient. Note that it is not appropriate to directly apply schemes that were derived for deterministic ordinary differential equations to (4) since the additional stochastic corrections from the Ito calculus must be included. For instance, it has been shown in [10] that the Euler method applied to a stochastic ordinary differential equation has a strong (pathwise) order of convergence of $\frac{1}{2}$ rather than order 1 as in the deterministic setting. Thus, it is essential to use schemes that are derived directly from suitable truncations of Taylor-Ito series. However, such schemes typically include derivatives of the drift and the diffusion coefficients. Given the highly nonlinear nature of these terms in (1), it is especially useful to consider derivative-free Runge-Kutta type schemes [10]. One choice of a first order strong scheme is the following:

$$
u_{n+1}=u_{n}+a\left(u_{n}\right) \Delta t+b\left(u_{n}\right) \Delta W+\frac{1}{2 \sqrt{\Delta t}}\left[b\left(\tilde{u}_{n}\right)-b\left(u_{n}\right)\right]\left[(\Delta W)^{2}-\Delta t\right]
$$

with supporting value $\tilde{u}_{n}$ given by

$$
\tilde{u}_{n}=u_{n}+b\left(u_{n}\right) \sqrt{\Delta t}
$$

and $\Delta W$ is a Gaussian with mean 0 and variance $\Delta t$. In practice, the time stepping is usually done in Fourier space so that terms, such as the standard diffusion, which are linear in Fourier space may be treated exactly through the use of an integrating factor. The integrating factor also allows the use of larger times steps than are numerically stable if the linear terms were not calculated exactly. Of course, since the solution of (1) is real, the $\Delta W_{n}$ 's in the time stepping scheme (when taking time steps in Fourier space) must be selected to respect the symmetries in Fourier coefficients that are present in spectral representations of real fields.

\section{Simulation Results}

In this section, some simulation results for (1) in two space dimensions using the numerical scheme described in the preceding section are given. The migration potential is chosen to be

$$
J_{m}(r)=\frac{1}{\sqrt{\pi r_{0}^{2}}} \exp \left(\frac{-|r|^{2}}{r_{0}^{2}}\right)
$$

with $r_{0}$ a parameter that describes the interaction length. The other parameters in (1) are chosen to be $D=0.1, \beta=6$, and $\gamma=r_{0}^{2}$. Simulation parameters include 64 wave numbers in each direction and a step size of $\Delta t=0.00001$, which insures numerical stability of the time evolver. The computational domain is a unit square with periodic boundary conditions. The system is initialized with two circular regions of high concentration surrounded by regions of low concentration. A rapidly decaying exponential is used to "connect" these two regions to 
insure the continuity and differentiability of the initial concentration field. The centers of the circles are a distance 0.5 apart. The upper circle initially has a radius of 0.1 , while the lower circle has an initial radius of 0.09 . This sort of initialization is commonly used in studies of Ostwald ripening as it is the simplest physical case in which this phenomenon can be observed ([11], [12]). In this system, the larger (upper) circle is expected to grow while the smaller (lower) circle shrinks since lower curvatures are preferred; as the smaller circle disappears, the larger region should be distorted away from its circular shape in the locations nearest the small circle but eventually become circular again. The deformation in shape would be expected to occur at both ends of the upper circle in this case due to the symmetries in the initial state and the periodic boundary conditions used.

Figure 1 contains contour plots from simulations of (1) using the spectral scheme described in Section 3 for the case $r_{0}=0.05$ for times $5,10,13.5$, and 15. The light areas represent regions of high concentration while the dark areas represent regions of low concentration. At the earlier times presented in Figure 1,
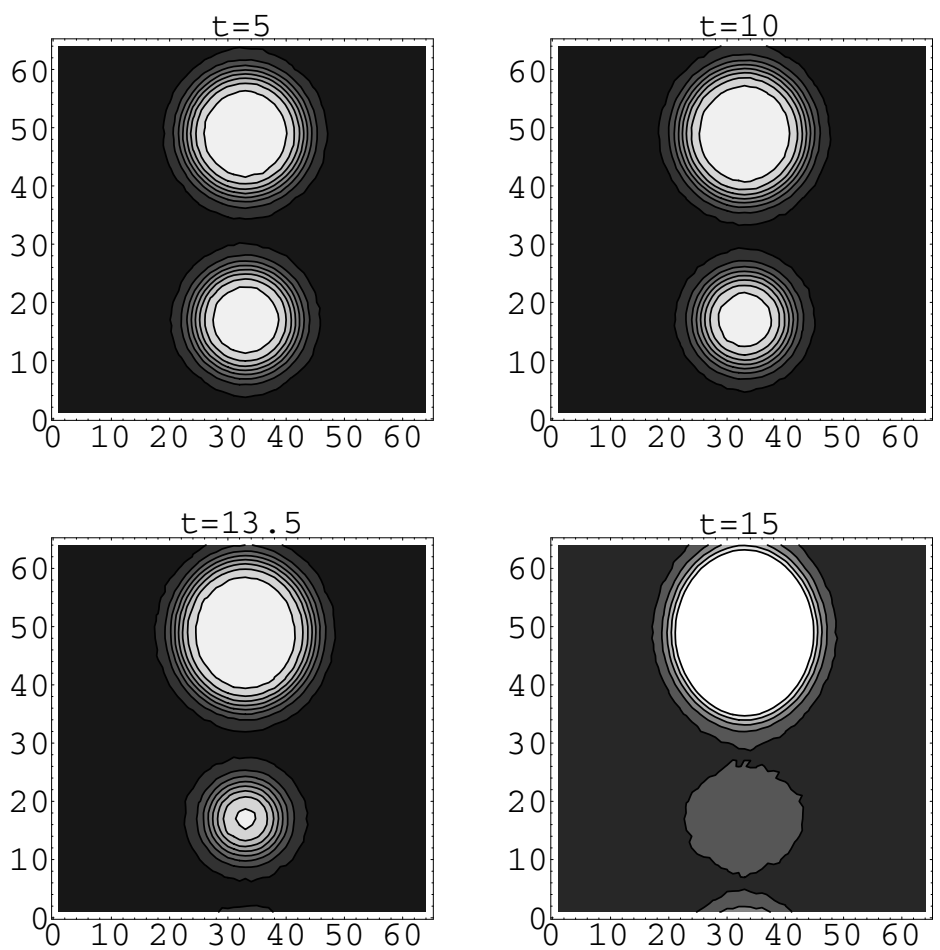

Fig. 1. Contour plots showing the time evolution of the concentration field obtained from mesoscopic simulations. The lighter shades represent regions of higher concentration while the darker shades represent regions of lower concentration. The expected Ostwald ripening is observed 
the shrinking of the lower circle and the growth of the upper circle are already evident. As time continues, the rate of shrinking of the smaller circle increases until at time 13.5, the region of highest concentration has almost completely disappeared from the lower circle; the deformation of the upper region into an elliptical shape is also becoming quite evident. By time 15, the lower region has essentially diffused away with the majority of particles appearing in the upper region which has become quite elliptical in shape. In later time results not shown here, this upper region eventually evolves to a circular shape again. The total concentration in the system was observed to be conserved for all times considered, including this final state. All of these results are in qualitative agreement with those expected; in addition, these results are also quite similar in nature those obtained in prior studies of others using different mathematical models and numerical techniques, for example, boundary integral techniques are used in $[12]$.

The ease of changing the interparticle interaction potential in the mesoscopic model in (1) straightforwardly allows for many computational studies. The effect
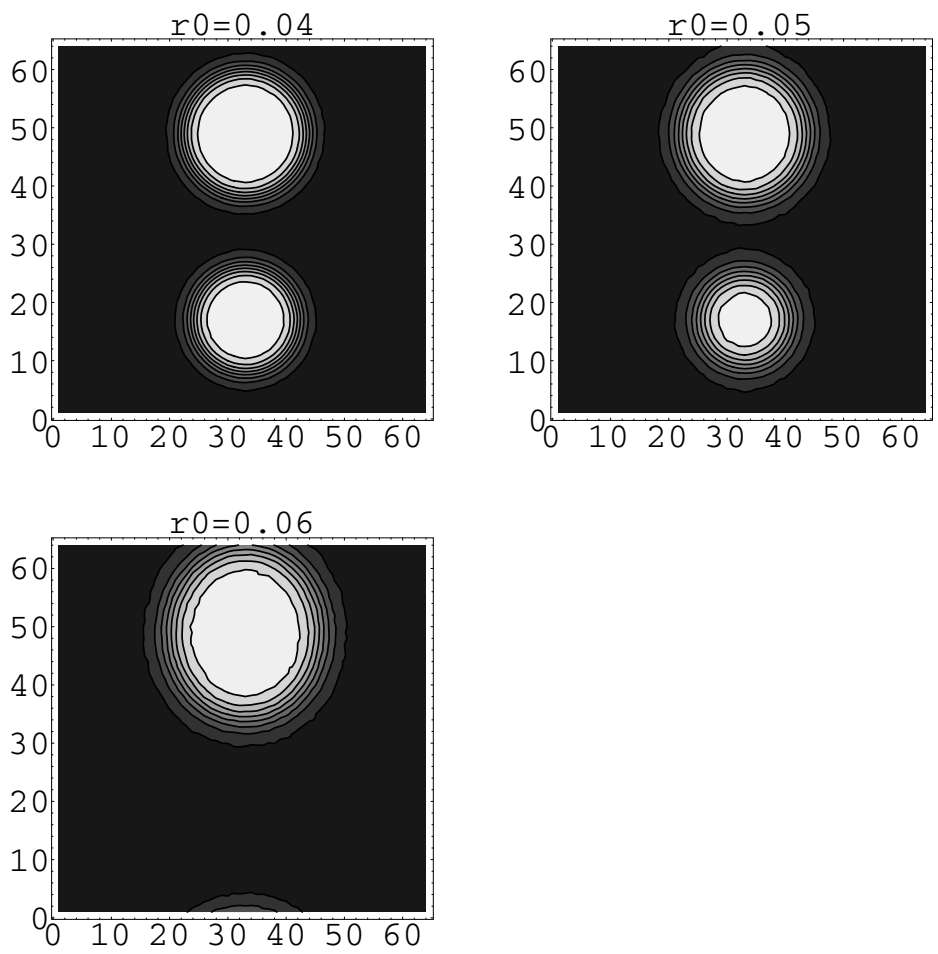

Fig. 2. Mesoscopic simulation results at time $t=10$ which demonstrate the effect of adjusting the particle interaction length on the time evolution of the system. Longer interaction lengths lead to faster evolution while shorter interaction lengths lead to slower evolution 
of adjusting the interaction length $r_{0}$ in (7) can be observed in Figure 2 where simulation results are given at time $t=10$ for $r_{0}=0.04,0.05$, and 0.06. All other simulation parameters are unchanged from those used for Figure 1; the initial concentration field is also unchanged. Recall that increasing the value of $r_{0}$ increases the interaction length of the underlying particles of the system. For the smallest interaction length shown here $\left(r_{0}=0.04\right)$, the system has evolved very little from the initial state; on the other hand, for the largest interaction radius shown here $\left(r_{0}=0.06\right)$, not only has the smaller (lower) circle completely diffused away but also the upper region has already evolved back to a nearly circular shape. These results are in good agreement with physical intuition since one would expect particles in systems with longer range interactions to more readily "find" each other. In fact, the smaller circle has essentially diffused away by time 8 when $r_{0}=0.06$ but remains until time 26 for the much smaller interaction radius of $r_{0}=0.04$. Also, just as for the simulation results in Figure 1, the total concentration was conserved throughout the entire simulation. Thus, the time scale of the evolution is quite sensitive to the interaction length of the particles but the overall qualitative behavior of the evolution remains the same.

\section{Conclusions}

This brief note can only indicate the value and power of using the mesoscopic modeling approach in conjunction with the spectral scheme for stochastic partial differential equations ("mesoscopic simulation"). For instance, mesoscopic simulation can be applied to physical situations with underlying micromechanisms other than diffusion. Mesoscopic simulation is more computationally tractable than other approaches such as molecular dynamics, especially for results which require long time simulations with large numbers of particles. (All results presented or discussed here were obtained in a few hours on a workstation.) One particularly attractive feature of this approach is the ease by which different sorts of particle interactions can be used. While Figure 2 certainly indicates one interesting class of adjustments to the interaction potential that can be considered, there are many others that should be considered including interactions with repulsive interaction ranges as might be encountered with charged particles. Anisotropic potentials would be useful in studies of coarsening systems in which there is a preferred growth direction. It is also important that more quantitative comparisons with theory and experiments be made. As a step in this direction, results of this type are given in [13], including simulations starting from a disordered state which exhibit the Lifshitz-Slyozov growth law as self-organization proceeds.

\section{Acknowledgments}

This research is partly supported by NSF-DMS-0219211, NSF-DMS-0406633, and an NJIT SBR grant. 


\section{References}

1. Voorhees, P.: The theory of Ostwald ripening. J. Statis. Phys. 38 (1985) 231-252.

2. Yao, J., Elder, K., Guo, H., Grant, M.: Late stage droplet growth. Physica A 204 (1994) $770-788$.

3. Niethammer, B., Pego, R.: The LSW model for domain coarsening: asymptotic behavior for conserved total mass. J. Statis. Phys. 104 (2001) 1113-1144.

4. Hildebrand, B., Mikhailov, A.: Mesoscopic modeling in the kinetic theory of adsorbates. J. Phys. Chem. 100 (1996) 19089-19101.

5. Giacomin, G., Lebowitz, J.: Exact macroscopic description of phase segregation in model alloys with long range interactions. Phys. Rev. Letters 76 (1996) 1094-1097.

6. Vlachos, D., Katsoulakis, M.: Derivation and validation of mesoscopic theories for diffusion-reaction of interacting molecules. Phys. Rev. Letters 85 (2000) 3898-3901.

7. Horntrop, D.: Spectral schemes for stochastic partial differential equations. (submitted).

8. Elliott, F., Horntrop, D., Majda, A.: Monte Carlo methods for turbulent tracers with long range and fractal random velocity fields. Chaos 7 (1997) 39-48.

9. Elliott, F., Horntrop, D., Majda, A.: A Fourier-wavelet Monte Carlo method for fractal random fields. J. Comp. Phys. 132 (1997) 384-408.

10. Kloeden, P., Platen, E.: Numerical Solution of Stochastic Differential Equations. Springer, Berlin (1992).

11. Imaeda, T., Kawasaki, K.: Theory of morphological evolution in Ostwald ripening. Physica A 186 (1992) 359-387.

12. Voorhees, P., McFadden, G., Boisvert, R., Meiron, D.: Numerical simulation of morphological development during Ostwald ripening. Acta Metall. 36 (1988) 207222.

13. Horntrop, D.: Mesoscopic simulation for domain coarsening. (in preparation). 